\title{
Steroid 21-hydroxylase Deficiency in a Newborn Female with Ambiguous Genitalia in Upper Egypt \\ AE Ahmed ${ }^{1}$, MH Hassan ${ }^{2}$
}

\begin{abstract}
Congenital adrenal hyperplasia "CAH" is a group of autosomal recessive disorders resulting from the deficiency of one of the five enzymes required for the synthesis of cortisol in the adrenal cortex. The most frequent is steroid 21-hydroxylase deficiency, accounting for more than 90 percent of cases and can have diverse manifestations: from the salt-wasting to the non-classical form due to a highly variable genetic mutation. We report a female infant aged two months, from Nag-Hammadi- Upper Egypt, whose presentation and laboratory abnormalities were consistent with $\mathrm{CAH}$ - classic 21-hydroxylase deficiency (salt wasting type). The patient presented with ambiguous genitalia and salt wasting. The karyotyping was that of normal female. There were elevated levels of serum 17-hydroxy progesterone, ACTH, rennin, androstenedione, and testosterone. Also, there was hyperkalemia and hyponatremia. We conclude that $\mathrm{CAH}$ to be keep in mind in the differential diagnosis of any newborn female with ambiguous genitalia with normal karyotyping and we call for dried blood spot 17-hydroxyprogesterone assay to be included in the newborn screening program in Egypt.
\end{abstract}

Keywords: Ambiguous genitalia, congenital adrenal hyperplasia, salt wasting, steroid 21hydroxylase, Upper Egypt

From: ${ }^{1}$ Department of Pediatrics, Faculty of Medicine, South Valley University, P. O. Box No. 83523 Qena, Egypt, ${ }^{2}$ Department of Biochemistry, Faculty of Medicine, South Valley University, P.O. Box No. 83523 Qena, Egypt

Correspondence: Dr MH Hassan, Department of Biochemistry and Molecular Biology Faculty of Medicine, South Valley University, Qena, Egypt.

E-mail: Mohammedhosnyhassaan@yahoo.com 


\section{INTRODUCTION}

Congenital adrenal hyperplasia "CAH" is a group of autosomal recessive disorders resulting from the deficiency of one of the five enzymes required for the synthesis of cortisol in the adrenal cortex. The most frequent is steroid 21-hydroxylase deficiency, accounting for more than 90 percent of cases and can have diverse manifestations: from the salt-wasting to the non-classical form due to a highly variable genetic mutation (1-2).

In $\mathrm{CAH}$, there are various genetic mutations in the enzymes involved in steroidogenesis. Due to this enzymatic defect, cortisol is under-produced and the negative feedback control on ACTH is lost with consequently excess ACTH produced in order to normalize cortisol levels resulting in overproduction and accumulation of steroids precursors prior to the enzyme defect as well as hyperplasia of the adrenal cortex. The clinical manifestation depends on the level of enzyme block in the steroid synthesis, see figure (1).

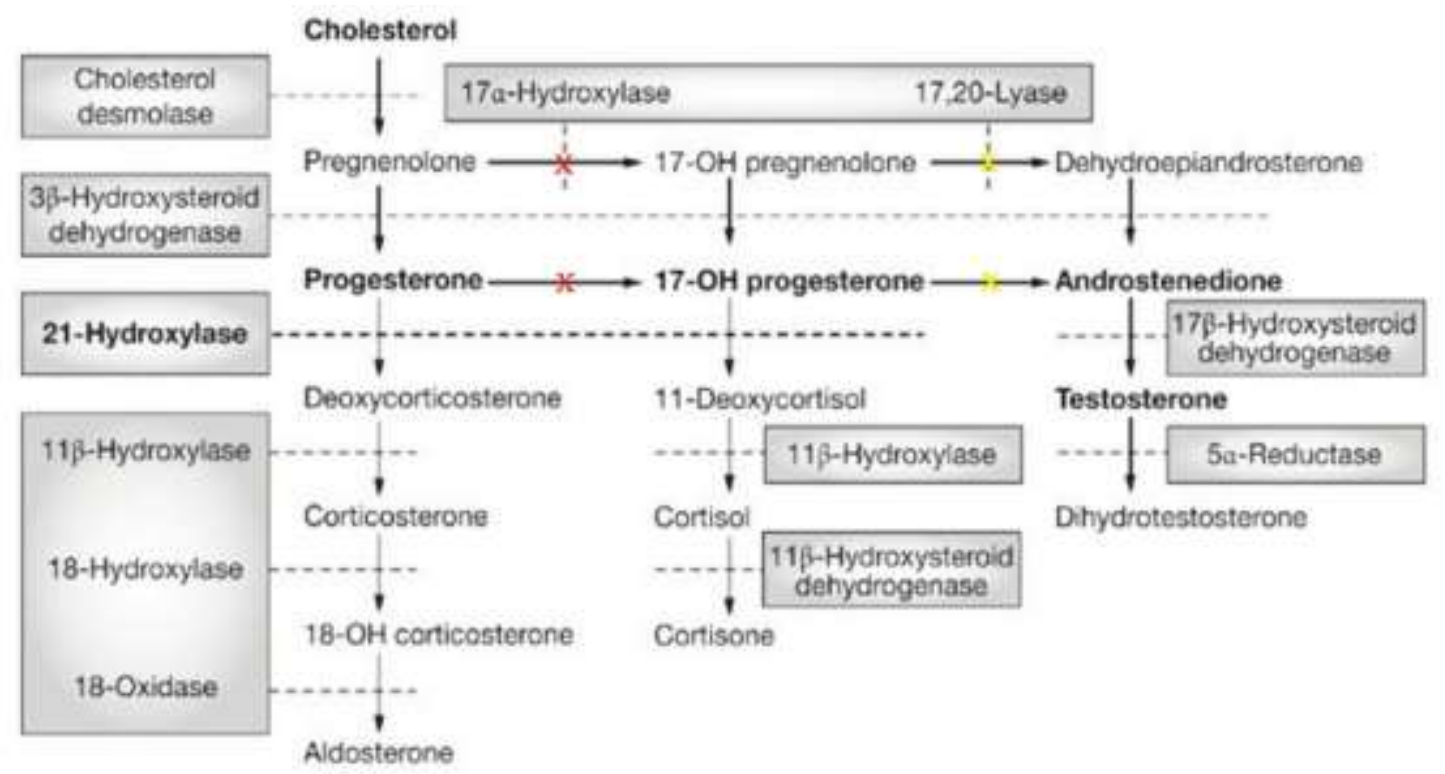

Fig. 1: Major human steroidogenic pathway in the adrenal cortex. Key enzymes are shown as dashed arrows/shaded boxes indicating the chemical reactions. P450scc (cholesterol desmolase) cleaves cholesterol to pregnenolone, the first intermediate in steroid biosynthesis. 
The steroids in the first row are $\Delta 5$-steroids, which constitute the preferred pathway to $\mathrm{C} 19$ steroids in human. Not all intermediate steroids, pathways, and enzymes are shown. In CAH due to $17 \alpha$-hydroxylase deficiency (marked as red $\mathrm{X}$ ) there is overproduction of the precursors/hormones on the left side of the first column with decreased production of the precursors/hormones on the right side of the second and third columns (3).

$\mathrm{CAH}$ should be considered in infants, children or adolescents with ambiguous genitalia, sexual infantilism, hypogonadism or hypertension, particularly when associated with disturbed water, electrolytes and hydrogen homeostasis. The most common form is $21 \alpha-$ hydroxylase deficiency which may be diagnosed at birth by the presence of virilization in female infants or by features of salt wasting in both genders (4). 11ß-hydroxylase deficiency is uncommon and $17 \alpha$-hydroxylase deficiency is a rare form of $\mathrm{CAH}$ which may present much later in adolescence or adulthood $(5,6)$.

\section{CASE REPORT}

A full-term baby aged 5 days (anterior fontanel: $5 \mathrm{~cm}$, head circumference: $36 \mathrm{~cm}$, chest circumference: $35 \mathrm{~cm}$, weight: $3,500 \mathrm{gm}$, length: $52 \mathrm{~cm}$ ) with abnormal external genitalia was born in a private hospital at Nag-Hammadi city-Qena- Upper Egypt. The baby was named Emad by his parents and was registered as a boy. By the $5^{\text {th }}$ day of the baby's life, the parents noticed significant yellow discoloration of the skin and sclera and they asked medical advice. When the treating physician examines baby's genitalia, he told the parents that the baby has hypospadius and the decision of the physician after doing serum bilirubin (Total: $17 \mathrm{mg} / \mathrm{dl}$, direct: $1.2 \mathrm{mg} / \mathrm{dl}$, indirect: $15.8 \mathrm{mg} / \mathrm{dl}$ ) was to be admitted at the neonatal intensive care unit "NICU" for receiving phototherapy and after discharge from the NICU, they should refer their baby to a urologist. 
At the NICU of Qena University Hospital- South Valley University, we examine the genitalia of the baby which was ambiguous with no palpable testes. Abdominal and pelvic ultrasound was done which reveals: a penis like structure (enlarged clitoris) is seen at the perineal region with no definite connection is seen with the urethra with no testes could be detected, but there was a normal sized uterus and ovaries. The result of the pelvic ultrasound direct us to do peripheral karyotype to exclude chromosomal abnormalities that lead to intersex which reveals 46, XX (normal female). The mother had never been treated with any drugs during pregnancy or encountered hormonal exposure in utero and she had no signs of androgen excess such as hirsutism, alopecia or clitoral hypertrophy (maternal virilization). There was no family history of infertility, ambiguous genitalia or unexplained neonatal death. The baby's name was changed after discharge from the NICU to Lila "female name".

Summary of the results of the biochemical laboratory parameters that have been measured in this case showed in table (1) that confirm the diagnosis of CAH- classic 21-hydroxylase deficiency (salt wasting type) at age of 2 months. Venous blood $(5 \mathrm{ml})$ was drawn and serum was separated by centrifugation at $3500 \mathrm{rpm}$ for $15 \mathrm{~min}$. We assay $\mathrm{pH}$ and serum electrolytes "sodium and potassium" using Easylyte Medica- USA. We measure the serum endocrinal panel required for diagnosis as shown in table (1) using Abbott Architect-USA. The infant is now on cortisol and aldosterone replacement therapy with regular follow up of serum sodium and potassium.

\section{DISCUSSION}

Steroid 21-hydroxylase (CYP21, also termed CYP21A2 and P450c21) is a cytochromeP-450 enzyme located in the endoplasmic reticulum. It catalyzes the conversion of17hydroxyprogesterone to 11-deoxycortisol, a precursor of cortisol, and the conversion of 
progesterone to deoxycorticosterone, a precursor of aldosterone. Owing to this loss of enzyme function, patients with 21-hydroxylase deficiency cannot synthesize cortisol efficiently, and as a result, the adrenal cortex is stimulated by corticotropin and overproduces cortisol precursors. Some of these precursors are diverted to the biosynthesis of sex hormones, which may cause signs of androgen excess, including ambiguous genitalia in newborn girls and rapid postnatal growth in both sexes. Concomitant aldosterone deficiency may lead to salt wasting with consequent failure to thrive, hypovolemia, and shock (1).

A spectrum of phenotypes is observed. A severe form with a concurrent defect in aldosterone biosynthesis (salt-wasting type) and a form with apparently normal aldosterone biosynthesis (simple virilizing type) are together termed classic 21 -hydroxylase deficiency. There is also a mild, nonclassic form that may be asymptomatic or associated with signs of postnatal androgen excess (2).

The present case was diagnosed as a classic 21-hydroxylase deficiency (salt wasting type) according to the clinical and biochemical findings. Clinically she had ambiguous genitalia in the form of a large clitoris, rugated and partially fused labia majora and a common urogenital sinus in place of a separate urethra and vagina as shown in figure (2). Biochemically, the karyotype is that of normal female in addition to other biochemical abnormalities summarized in table (1) in the form of high serum level of 17hydroxyprogesterone, progesterone, estradiol "E2", ACTH, rennin, androstenedione, total testosterone, acidosis, low cortisol and aldosterone levels with hyperkalemia and hyponatremia. 
Table 1: Abnormal biochemical findings in the studied case

\begin{tabular}{llll}
\hline $\begin{array}{l}\text { Biochemical } \\
\text { parameters }\end{array}$ & Result & Reference range & Interpretation \\
\hline Cortisol & $2.6 \mu \mathrm{g} \backslash \mathrm{dl}$ & $3-16.6$ & Decreased \\
Aldosterone & $4.2 \mathrm{mg} / \mathrm{dl}$ & $5.8-110$ & Decreased \\
ACTH & $42 \mathrm{pg} / \mathrm{ml}$ & up to 30 & Increased \\
Progesterone & $19.3 \mathrm{ng} / \mathrm{ml}$ & $0.87-3.37$ & Increased \\
17-hydroxy progesterone & $5 \mathrm{ng} / \mathrm{ml}$ & $0.5-2.4$ & Increased \\
Estradiol "E2" & $17 \mathrm{pg} / \mathrm{ml}$ & $<10$ & Increased \\
Sodium & $121.8 \mathrm{mEq} / 1$ & $135-155$ & Hyponatremia \\
Potassium & $6.5 \mathrm{mEq} / 1$ & $3.6-5.5$ & Hyperkalemia \\
pH & 7.1 & $7.35-7.45$ & Acidosis \\
Testosterone (total) & $0.71 \mathrm{ng} / \mathrm{ml}$ & $.1-0.56$ & Increased \\
Androstenedione & $0.9 \mathrm{ng} / \mathrm{ml}$ & up to 0.5 & Increased \\
Renin & $6.7 \mathrm{ng} / \mathrm{ml} / \mathrm{hr}$ & $2.4-3.7$ & Increased \\
\hline
\end{tabular}
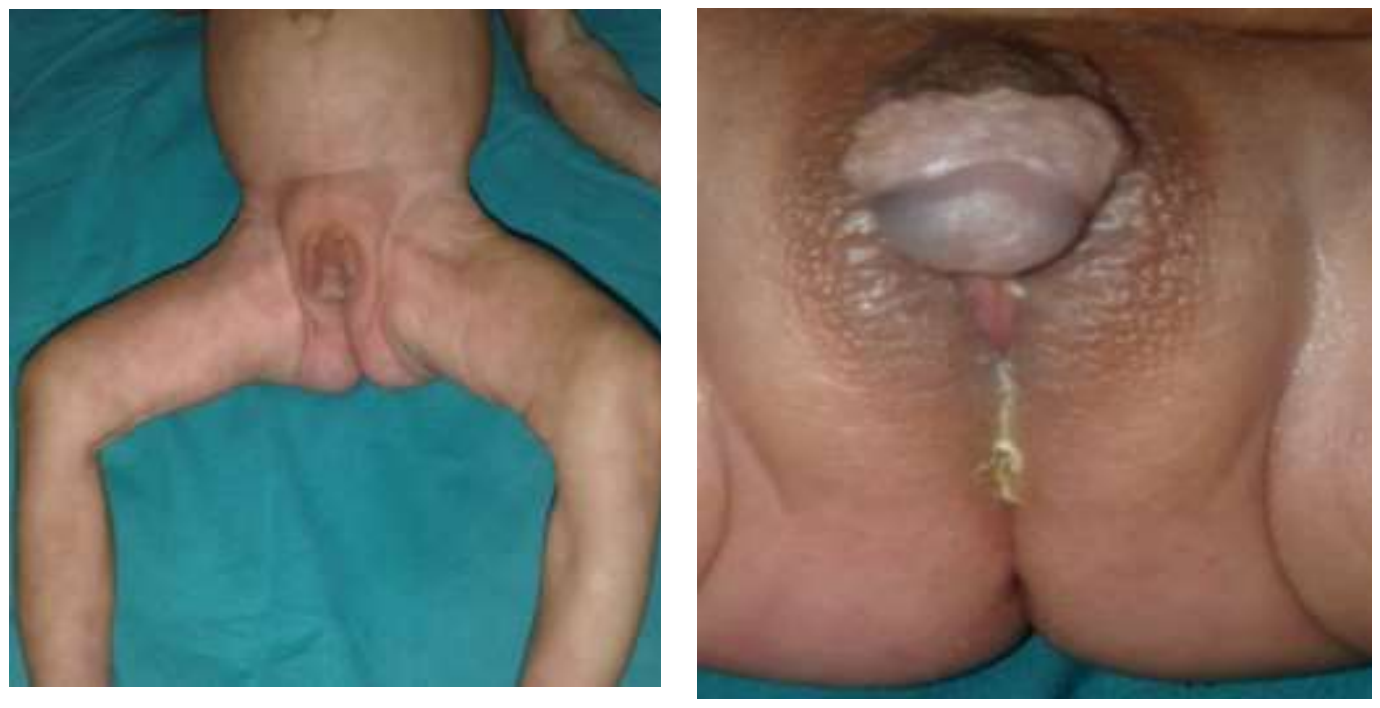

Fig. 2: Female newborn with congenital adrenal hyperplasia with ambiguous genitalia: a large clitoris, rugated and partially fused labia majora and a common urogenital sinus in place of a separate urethra and vagina.

Approximately 75 percent of patients with classic 21-hydroxylase deficiency have severely impaired 21-hydroxylation of progesterone and thus cannot adequately synthesize aldosterone. Elevated levels of 21-hydroxylase precursors (progesterone and 17hydroxyprogesterone) may act as mineralocorticoid antagonists, exacerbating the effects of 
aldosterone deficiency (7). Since aldosterone regulates sodium homeostasis, renal sodium excretion in untreated patients is excessive and can result in hypovolemia and hyperreninemia. Such patients cannot excrete potassium efficiently and are prone to hyperkalemia, especiallyin infancy. Cortisol deficiency in these patients contributes to poor cardiac function, poor vascular response to catecholamines, a decreased glomerular filtration rate, and increased secretion of antidiuretic hormone (8). Thus, cortisol and aldosterone deficiency together cause hyponatremic dehydration and shock in inadequately treated patients (9).

Patients with the salt-wasting form are identified through the measurement of serum electrolytes, aldosterone, and plasma renin and the finding of expected abnormalities: hyperkalemia, low levels of aldosterone, and hyperreninemia. Age-specific ref-erence values for renin should be used, since plasma renin activity is normally higher in neonates than in older children (10). In the classic forms, epinephrine deficiency occurs because adrenocortical secretion of cortisol is necessary for adrenomedullary organogenesis and adrenomedullary epinephrine synthesis (11). Both cortisol and epinephrine are counterregulatory hormones and play an essential role in the regulation of blood glucose which explains the hypoglycemia occurring in patients with $\mathrm{CAH}$ especially during stress conditions (12).

Treatment in congenital adrenal hyperplasia should aim to ensure normal growth in infancy and childhood, the development of puberty at the appropriate age and later, the acquisition of adult reproductive potential (13). The standard treatment for classic CAH due to 21-hydroxylase deficiency is glucocorticoid (replaces cortisol) and fludrocortisone (replaces aldosterone), given daily. Glucose supplementation is also warranted to prevent hypoglycemia (12). It is essential for the infant to be seen early by a surgeon experienced inthe techniques required for reconstruction of the genitalia. There are basically two 
structural abnormalities which require surgical treatment: reduction in the size of the enlarged clitoris and division of the fused labial folds to exterior rise the vaginal opening (13).

In conclusion, any newborn female infant with ambiguous genitalia should be considered as a medical emergency and should be investigated as soon as possible as this problem cause psychic trauma to the parents and to the infant itself as she growing up because of the incorrect gender assignment. It is important to keep in mind that $\mathrm{CAH}$ is one of the important causes of ambiguous genitalia of the newborn and the commonest cause of congenital adrenal hyperplasia is 21-hydroxylase deficiency. Assay of serum $17 \mathrm{OH}$ progesterone concentration and determination of a peripheral karyotype are essential for diagnosis.

\section{AUTHORS' NOTE}

The authors report no conflicts of interest in this work. 


\section{REFERENCES}

1. Speiser PW, White PC. Congenital adrenal hyperplasia. N Engl J Med 2003; 349: 776-88.

2. White PC, Speiser PW. Congenital adrenal hyperplasia due to 21-hy-droxylase deficiency. Endocr Rev 2000; 21: 245-91.

3. Nimkarn S, New M. Prenatal diagnosis and treatment of congenital adrenal hyperplasia owing to 21-hydroxylase deficiency. Nat Clin Pract Endocrinol Metab $2007 ; 3(5): 405-413$.

4. Krone N, Arlt W. Genetics of congenital adrenal hyperplasia. Best Pract Res Clin Endocrinol Metab 2009; 23(2):181-192.

5. Karter CE, Biglieri EG. Disorders of steroid $17 \alpha$-hydroxylase deciency. Endocrinol Metab North Am 1994; 23(2):341-357.

6. Miller WL, Auchus RJ. E molecular biology, biochemistry, and physiology of human steroidogenesis and its disorders. Endocr Rev 2011; 32(1):81-151.

7. Oelkers WK. Effects of estrogens andprogestogens on the renin-aldosterone system and blood pressure. Steroids 1996; 61:166-71.

8. Lamberts SW, Bruining HA, de Jong FH.Corticosteroid therapy in severe illness.N Engl J Med 1997; 337:1285-92.

9. Merke DP, Chrousos GP, Eisenhofer G,et al. Adrenomedullary dysplasia and hypofunction in patients with classic 21-hydroxy-lase deficiency. N Engl J Med 2000; 343:1362-8.

10. Fukushige J, Simomura K, Ueda K. Influence of upright activity on plasma rennin activity and aldosterone concentration in children. Eur J Pediatr 1994; 153:284-6. 
11. Merke DP, Chrousos GP, Eisenhofer G, Weise M, Keil MF, Rogol AD, et al. Adrenomedullary dysplasia and hypofunction in patients with classic 21-hydroxylase deficiency. N Engl J Med 2000; 343(19): 1362-1368.

12. Keil MF, Bosmans C, Ryzin CV, Merke D. Hypoglycemia during acute illness in children with classic congenital adrenal hyperplasia. J Pediatr Nurs. 2010; 25(1): 18.

13. Puri N, Talwar A. A Case Report of Classic Adrenal Hyperplasia - A Rarity. JIMSA 2014; 27 (1): 51-52. 